\title{
Effect of Heart Failure on Acute Ischemic Stroke Severity
}

\author{
Taha Kamel Alloush', Mahmoud Haroun Ibrahim1, Nahed Salah El-Dein Ahmed1, \\ Ghada Samir El-Shahed"1, Lobna M. El-Nabil El-Sayed1*, Mohamed Hamdy Ibrahim¹, \\ Hosam Ahmed Azmy² \\ ${ }^{1}$ Faculty of Medicine, Ain Shams University, Cairo, Egypt \\ ${ }^{2}$ Ministry of Health, Cairo, Egypt \\ Email: ${ }^{*}$ mohamedhamdy_neuro2007@yahoo.com
}

Received 9 June 2014; revised 9 July 2014; accepted 8 August 2014

Copyright (C) 2014 by authors and Scientific Research Publishing Inc.

This work is licensed under the Creative Commons Attribution International License (CC BY). http://creativecommons.org/licenses/by/4.0/

(c) (i) Open Access

\begin{abstract}
Objectives: To assess the impact of heart failure diseases on stroke severity and short term (1 month mortality). Materials and Methods: Totally, 200 patients admitted to Ain Shams university specialized hospital, were diagnosed clinically to have acute ischemic stroke within 3 days. History taking about previous heart disease was taken, and full general and neurological examinations were done. Full metabolic profile, cardiac examination and investigations were done. Carotid duplex, MRI stroke protocol and lastly clinical reevaluation using NIH Stroke Scale (NIHSS) after 1 month from stroke onset were done. Results: Patients with systolic dysfunction (EF $\leq 40 \%$ ) had lacunar infarction in $18.75 \%$ of cases, partial anterior circulation infarction in $62.5 \%$ of cases, posterior circulation infarction in $6.25 \%$ of cases and total anterior circulation infarction in $12.5 \%$ of cases. Leucoaraiosis was present in $87 \%$ of cases and significant intracranial vessel stenosis was showed in $\mathbf{8 7 . 5 \%}$ who had done MRI. Their median NIHSS score at admission was 10.5 with IQR of 5 - 21 and at follow-up it was 5 with IQR of 2.5 - 10.5. At follow-up $22.7 \%$ (5 patients) were dead. Conclusion: Systolic dysfunction ( $\mathrm{EF}<40)$ i.e. heart failure has no significant effect on stroke severity and 1 month follow up prognosis.
\end{abstract}

\section{Keywords}

Heart Failure, Stroke Prognosis, Ejection Fraction

\section{Introduction}

Stroke is a neurological emergency that requires rapid diagnostic workup and immediate treatment [1]. The de-

${ }^{*}$ Corresponding author.

How to cite this paper: Alloush, T.K., Ibrahim, M.H., El-Dein Ahmed, N.S., El-Shahed, G.S., El-Nabil El-Sayed, L.M., Ibrahim, M.H. and Azmy, H.A. (2014) Effect of Heart Failure on Acute Ischemic Stroke Severity. Open Journal of Medical Imaging, 4, 103-107. http://dx.doi.org/10.4236/ojmi.2014.43014 
finition of stroke for clinical trials has required either symptoms lasting more than 24 hours or imaging of an acute clinically relevant brain lesion in patients with rapidly vanishing symptoms. The use of the term "brain attack" which may lack specificity has been championed by the educational campaigns of national health organizations to help inform the public about the urgency of stroke [2]. Cardiac sources of embolism has been estimated to be etiologic factor in up to $20 \%$ of ischemic stroke cases. Although there are a large number of cardiac diseases reported to be a potential source of embolism to the brain, the majority of causes can be diagnosed by straightforward clinical examination, electrocardiography, chest X-ray along with non-invasive echocardiography [3]. Among these numerous causes, some are much more threatening as prosthetic valves and rheumatic atrial fibrillation than others like mitral leaflet prolapse and patent foramen oval [4]. Different cardiac diseases have their own impact on severity, subtyping and prognosis of stroke [5].

\section{Subjects and Methods}

A total of 200 patients admitted to Ain Shams university specialized hospital, stroke unit were recruited to this study between July 2009 and March 2011, diagnosed clinically to have acute ischemic stroke within 3 days. Ethical committee of Ain Shams University Specialized Hospital, Cairo, Egypt had approved the clinical study. The patient, patient family and party were informed orally about objectives, risk factor and benefit of the study. And verbal approvals, consent were obtained and taken.

\subsection{Inclusion Criteria}

Patients were diagnosed clinically to have ischemic stroke if they had "rapidly developing clinical symptoms and/or signs of focal, and at times global, disturbances of cerebral function, lasting more than 24 hours or leading to death with no apparent cause other than that of vascular origin” (Hatano, 1976), CT or Gradient echo MRI does not show areas of haemorrhage.

\subsection{Exclusion Criteria}

Patients with haemorrhagic infarction.

\subsection{Methods}

Patients who fulfilled the above criteria were subjected to the following: Clinical evaluation: This was done within the first 3 days of admission by history taking general and neurological examination and stroke severity assessment using the national institute of health stroke scale (NIHSS) and then stroke was subdividing according to modified oxfordshire classification into total anterior, partial anterior, lacunar and posterior circulation stroke as proposed by (Lee et al., 2000) [6]. This stroke subtyping was then correlated with diffusion-weighted MRI and if there is any conflict between clinical and radiological findings, diffusion-weighted imaging was taken as decisive. History was also taken for: Hypertension, Diabetes mellitus, History of ischemic heart disease (history of myocardial infarction, angina pectoris or the patient's reporting of a positive diagnostic test (stress ECG, coronary angiography) or drug treatment.

II-Routine investigations: These included complete blood picture, erythrocyte sedimentation rate, coagulation profile, random blood sugar, liver and kidney function tests, lipid profile, uric acid level, plain chest x-ray and electrocardiography (ECG).

III-Transthoracic Echocardiography: Where the following data were detected: Ejection fraction (EF), patients were considered to have heart failure or systolic dysfunction if ejection fraction is $\leq 40 \%$ [7].

IV-Carotid duplex: This was done using a $7.5 \mathrm{MHz}$ transducer on a Philips ultrasound machine, peak systolic and mean flow velocity were measured for the common carotid, internal carotid and external carotid arteries on both sides. Vertebral arteries were assessed when visualized. Significant carotid stenosis was defined as narrowing of the lumen of $\geq 70 \%[8]$.

V-MRI-Brain (stroke protocol; axial T1, T2, FLAIR, T2*, DWI and MRA).This was done using 1.5 tesla using a head coil, the examination lasted for 20 minutes according to Ain Shams protocol of stroke imaging sequencing which includes axial T1, T2, Flair, DWI, Gradient echo (T2*) and MRA (Three dimensional time of flight), Where the following data were obtained:

Size of the infarct and its arterial territory where infarcts of $\leq 1.5 \mathrm{~cm}$ where considered lacunar infarct, infarcts 
corresponding to the distribution of internal carotid or mainstem middle cerebral artery territory were considered total anterior circulation infarct and infarcts in the distribution of vertebrobasilar territory were considered posterior circulation infarct and after comparison with clinical classification, anatomical or modified oxfordshire classification was made [8]. Presence or absence of intra-cranial stenosis according to (WASID method), by comparing the diameter of the stenosed area and the diameter (D) of the proximal vessel just proximal to the stenosed vessel and applying the following equation:

Percent stenosis $=[1-($ Dstenosis/Dnormal) $] \times 100 \%$ where percentage $\geq 25 \%$ were taken as positive value [9]. Patients were followed up by NIHSS after 1 month.

\section{Statistical Analysis}

The data were coded, entered and processed on computer using SPSS (version 15). The level $\mathrm{P}<0.05$ was considered the cut-off value for significance. Chi-Square test $\mathrm{X}^{2}$ was used to test the association variables for categorical data. Fisher exact test was performed in table containing value less than 5 Student's t-test was used to assess the statistical significance of Statistical Analysis The data were coded, entered and processed on computer using SPSS (version 15). The level $\mathrm{P}<0.05$ was considered the cut-off value for significance. Chi-Square test $\mathrm{X}^{2}$ was used to test the association variables for categorical data. Fisher exact test was performed in table containing value less than 5 Student's t-test was used to assess the statistical significance of the difference between two population means in a study involving independent samples. The Wilcoxon signed-rank test to assess the statistical significance of the difference between two populations in a study involving matched or paired samples.

\section{Results}

Demographic data: This study included 200 patients, with an age range between 23 - 90 years with a mean and a standard deviation of $61.87 \pm 12.65$ of those 118 (59\%) patients were males and 82 (41\%) were females. Of our patients, $72 \%$ were hypertensive, $53 \%$ were diabetic, $37 \%$ gave a history of ischemic heart disease, $16.5 \%$ were in atrial fibrillation while 33\% were cigarette smokers. Stroke cases were classfied according to Oxfordshire classification in Table 1.

Full neurological examination was done using NIHSS to all patients at admission and one month later. All related data are mentioned in Table 2.

Table 1. Types of stroke according to modified oxfordshire classification.

\begin{tabular}{|c|c|c|c|}
\hline & \multicolumn{2}{|c|}{$\mathrm{N}(148)$} & \multirow{2}{*}{$\begin{array}{c}\% \\
34.4\end{array}$} \\
\hline \multirow{4}{*}{ Types } & Lacunar & 51 & \\
\hline & PACI & 65 & 43.9 \\
\hline & POCI & 25 & 16.2 \\
\hline & TACI & 7 & 4.7 \\
\hline \multirow{3}{*}{ Outcome } & & $\mathrm{N}(200)$ & $\%$ \\
\hline & Alive & 174 & 87 \\
\hline & Dead & 26 & 13 \\
\hline
\end{tabular}

PACI: Partial anterior circulation syndrome, POCI: Posterior circulation infarction, TACI: Total anterior circulation infarction.

Table 2. NIHSS at admission and at follow-up.

\begin{tabular}{|c|c|c|c|c|c|c|c|}
\hline & & & & & $\mathrm{Z}$ & $\mathrm{P}$ & \\
\hline & \multicolumn{2}{|c|}{ At admission (200) } & \multicolumn{2}{|c|}{ At follow-up (174) } & & & Sig. \\
\hline & Median & IQR & Median & IQR & & & \\
\hline NIHSS & 8 & $5-14$ & 4 & $2-8$ & 9.27 & $<0.0001$ & HS \\
\hline
\end{tabular}

Wilcoxon Signed Ranks Test, NIHSS: National institute of health stroke scale, IQR: Interquartile range. 
There was a highly statistical significant decrease in NIH score at follow up when compared to NIH at admission $\mathrm{P}<0.0001$. Echocardiography was done showed that systolic dysfunction represented by ejection fraction of $\leq 40 \%$ was seen in 22 patients (11\%). Patients with systolic dysfunction (EF $\leq 40 \%)$ had lacunar infarction in $18.75 \%$ of cases, partial anterior circulation infarction in $62.5 \%$ of cases, posterior circulation infarction in $6.25 \%$ of cases and total anterior circulation infarction in $12.5 \%$ of cases. Leucoaraiosis was present in $87 \%$ of cases and significant intracranial vessel stenosis in $87.5 \%$ who had done MRI. None of the cases who had done carotid duplex study (12 patient) had significant carotid stenosis, their median NIHSS score at admission was 10.5 with IQR of 5 - 21 and at follow-up was 5 with IQR of 2.5 - 10.5. At follow-up 22.7\% (5 patients) were dead (Table 3).

\section{Discussion}

Stroke is a major public health problem because it is frequent, dangerous and expensive. Moreover, it can often be prevented, and may now be treatable in the acute stage [10]. The incidence of stroke is now higher than that of acute coronary syndromes. Patients with incident strokes are the target for acute stroke management. Stroke is the most prevalent neurological disorder under the age of 85 years and $25 \%$ of males and $20 \%$ of females are expected to have stroke if they live up to this age. Patients with prevalent stroke and transient ischaemic attacks are the target for secondary prevention [11].

Heart failure with impaired systolic function was documented to be associated with severer stroke and increased incidence of long-term mortality and morbidity after ischemic stroke [12]. Heart failure patients are more prone to develop ischemic stroke and there is a steady association between ischemic stroke and heart failure of variable severity [13]. It was found that patients with Left ventricular ejection fraction (LVEF) of $28 \%$ or less had a relative risk of stroke of 1.86 compared with patients with LVEF of 35\% - 40\%. Also it was reported that left ventricular dysfunction with $\mathrm{EF}$ of $\leq 40 \%$ was more prevalent in patients who had embolic stroke [14].

In our study, heart failure patients with ejection fraction (EF) of $\leq 40 \%$ were only $11 \%$ of the whole sample with a mean NIHSS score at admission of 10.5 which is higher than those with ejection fraction of $>40 \%$ which was 8 but again this did not reach statistical significance. Also, it was not an independent risk factor by multivariate analysis and the explanation for this may be the fact that patients with systolic dysfunction were relatively

Table 3. Clinical data of patients with systolic dysfunction ( $\mathrm{EF} \leq 40 \%)$.

\begin{tabular}{|c|c|c|c|c|c|}
\hline & & $\mathrm{N}$ & $\%$ & $\mathrm{P}$ & Sig. \\
\hline \multirow{4}{*}{ Types (16) } & Lacunar & 3 & 18.75 & \multirow{4}{*}{0.07} & \multirow{4}{*}{ NS } \\
\hline & PACI & 10 & 62.5 & & \\
\hline & POCI & 1 & 6.25 & & \\
\hline & TACI & 2 & 12.5 & & \\
\hline \multirow[b]{2}{*}{ Leucoaraiosis (16) } & +ve & 14 & 87.5 & \multirow[b]{2}{*}{0.28} & \multirow[b]{2}{*}{ NS } \\
\hline & -ve & 2 & 12.5 & & \\
\hline \multirow{2}{*}{ Significant carotid stenosis (12) } & +ve & 0 & 0 & \multirow{2}{*}{0.09} & \multirow{2}{*}{ NS } \\
\hline & -ve & 12 & 100 & & \\
\hline \multirow{2}{*}{ Significant intracranial stenosis (16) } & $+\mathrm{ve}$ & 14 & 87.5 & \multirow[b]{2}{*}{0.45} & \multirow[b]{2}{*}{ NS } \\
\hline & -ve & 2 & 12.5 & & \\
\hline NIHSS at admission (22) & \multicolumn{3}{|c|}{10.5 (IQR 5 - 21) } & \multirow{2}{*}{0.02} & \multirow{2}{*}{$\mathrm{S}$} \\
\hline NIHSS at follow-up (17) & \multicolumn{3}{|c|}{5 (IQR 2.5 - 10.5) } & & \\
\hline \multirow{2}{*}{ Outcome (22) } & \multicolumn{2}{|l|}{ Alive } & \multicolumn{2}{|l|}{17} & \\
\hline & \multicolumn{2}{|l|}{ Dead } & \multicolumn{2}{|l|}{5} & \\
\hline
\end{tabular}

Fisher's Exact Test; EF: Ejection fraction, IQR: Interquartile range, NIHSS: National institute of health stroke scale PACI: Partial anterior circulation infarction; POCI: Posterior circulation infarction, TACI: Total anterior circulation infarction. 
small in number compared to the whole sample recruited. As a conclusion in the study we failed to prove the significant effect of Systolic dysfunction $(\mathrm{EF}<40)$ (i.e. heart failure) on stroke severity and 1 month follow up prognosis.

\section{Recommendation for Further Study}

We also recommend further work with larger numbers of patients having specific echocardiographic variables as DD, SWMA and stratification of the degree of impairment of certain variables as systolic dysfunction left atrial dilatation and systolic heart failure.

\section{References}

[1] Einhaupl, K.M., Villringer, A. and Meister, W. (1999) Heparin Treatment in Sinus Venous Thrombosis. Lancet, 338, 597-600. http://dx.doi.org/10.1016/0140-6736(91)90607-Q

[2] Sacco, R. (2009) No Significant Reduction in Progression of Symptomatic Intracranial Stenosis with Cilostazol versus Clopidogrel. European Stroke Conference (ESC), Medscape Today. http://www.medscape.com/viewarticle/704459

[3] Sandercock, P., Bamford, J., Dennis, M., et al. (1992) Atrial Fibrillation and Stroke: Prevalence in Different Types of Stroke and Influence on Early and Long Term Prognosis (Oxfordshire community stroke project). BMJ, 305, 14601465. http://dx.doi.org/10.1136/bmj.305.6867.1460

[4] Longmore, M., Wilkinson, I.B., Davidson, E.H., et al. (2010) Arrythmias. In: Oxford Handbook of Medicine, 8th Edition, Oxford University Press, Oxford, 118.

[5] José, B., Betsy, B. and Michael, J. (2008) Vascular Diseases of the Nervous System, In: Neurology in Clinical Practice, 5th Edition, Butterworth-Heinemann, Oxford, 1165.

[6] Lee, L.J., Kidwell, C.S., Alger, J., et al. (2000) Impact on Stroke Subtype Diagnosis of Early Diffusion-Weighted Magnetic Resonance Imaging and Magnetic Resonance Angiography. Stroke, 31, 1081-1089.

http://dx.doi.org/10.1161/01.STR.31.5.1081

[7] Carmine M, Federica S, Simona S, et al. (2005) Contribution of Atrial Fibrillation to Incidence and Outcome of Ischemic Stroke, Results from a Population-Based Study. Stroke, 36, 1115-1119. http://dx.doi.org/10.1161/01.STR.0000166053.83476.4a

[8] José, B., Betsy, B., Michael, J., et al. (2004) Vascular Diseases of the Nervous System. In: Neurology in Clinical Practice, 4th Edition, Butterworth-Heinemann, Oxford, 1197.

[9] Samuels, O.B., Joseph, G.J., Lynn, M.J., et al. (2000) A Standardized Method for Measuring Intracranial Arterial Stenosis. American Journal of Neuroradiology, 21, 643-646.

[10] Biller, J., Betsy, B. and Michael, J. (2008) Vascular Diseases of the Nervous System. In: Neurology in Clinical Practice, 5th Edition, 1165.

[11] Cordonnier, C. and Leys, D. (2008) Stroke, the Bare Essentials. Practical Neurology, 8, 263-272. http://dx.doi.org/10.1136/jnnp.2008.149401

[12] Pullicino, P.M., Halperin, J.L. and Thompson, J.L. (2000) Stroke in Patients with Heart Failure and Reduced Left Ventricular Ejection Fraction. Neurology, 54, 288. http://dx.doi.org/10.1212/WNL.54.2.288

[13] Allison, G., Ralph, L., Tanja, R., et al. (2006) Left Ventricular Systolic Dysfunction and the Risk of Ischemic Stroke in a Multiethnic Population. Stroke, 37, 1715-1719. http://dx.doi.org/10.1161/01.STR.0000227121.34717.40

[14] Park, D.C., Nam, H.S., Lim, S.R., et al. (2000) MRI Features of Infarcts with Potential Cardiac Source of Embolism in the Yonsei Stroke Registry (YSR), Korea. Yonsei Medical Journal, 4, 431. 
Scientific Research Publishing (SCIRP) is one of the largest Open Access journal publishers. It is currently publishing more than 200 open access, online, peer-reviewed journals covering a wide range of academic disciplines. SCIRP serves the worldwide academic communities and contributes to the progress and application of science with its publication.

Other selected journals from SCIRP are listed as below. Submit your manuscript to us via either submit@scirp.org or Online Submission Portal.
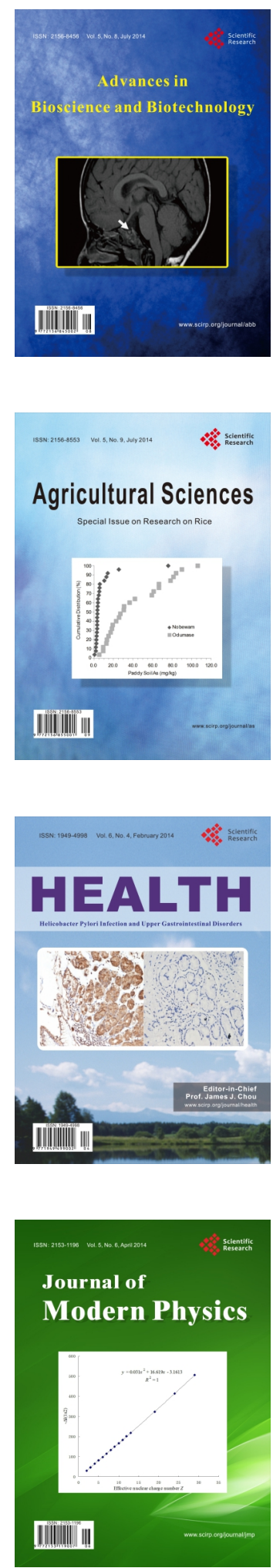
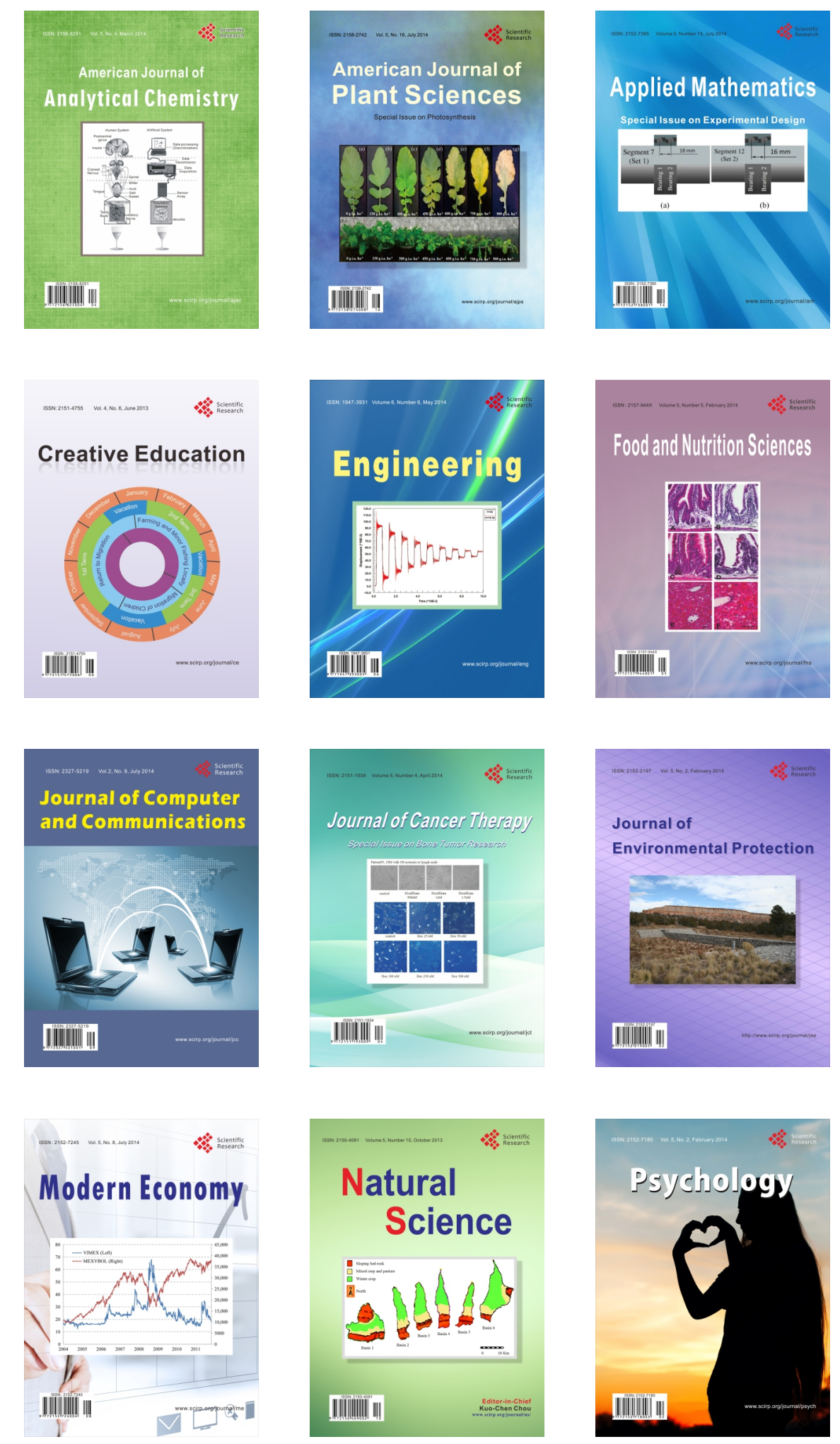